\title{
Funções reais de duas variáveis e GeoGebraBook: recursos dinâmicos para o ensino de Cálculo
}

\author{
Real functions of two variables and GeoGebraBook: dynamic means for the \\ teaching of Calculus
}

Raiane Lemke ${ }^{1}$

Ivanete Zuchi Siple ${ }^{2}$

\section{Resumo}

Fruto de uma pesquisa qualitativa de programa de Pós-Graduação em Ensino de Ciências, Matemática e Tecnologias da Universidade do Estado de Santa Catarina, este artigo apresenta um produto educacional sobre o Ensino de Cálculo, especialmente no que diz respeito ao ensino de funções reais de duas variáveis reais e alguns assuntos de derivadas parciais, como interpretação geométrica e taxa de variação. A partir de outras pesquisas constatou-se uma problemática no ensino e aprendizagem dos conteúdos supracitados. Nesse sentindo, considerando a questão da visualização, softwares de geometria dinâmica e nos embasando no quadro teórico do Conhecimento Tecnológico e Pedagógico do Conteúdo (TPACK), foi desenvolvido um produto educacional - um livro dinâmico construído na opção de livro no site do GeoGebra - GeoGebraBook, que contém propostas de exercícios, atividades, perguntas, imagens, vídeos, situaçõesproblemas e objetos de aprendizagem desenvolvidos no GeoGebra, organizados de maneira interativa, com o intuito de contribuir nos processos de ensino e aprendizagem dos conteúdos envolvidos.

Palavras-chave: Cálculo. GeoGebra. Funções reais de duas variáveis.

\section{Abstract}

As a result of qualitative research of the postgraduate program in Science, Mathematics and Technology Education of the State University of Santa Catarina, this article presents an educational product on the teaching of calculus, especially with respect to teaching real functions of two real variables and some subjects of partial derivatives, such as geometric interpretation and rate of change. Based on other research, there was a problem in the teaching and learning of the aforementioned contents. So considering the issue of visualization, dynamic geometry software, and based on the theoretical framework of the Technological Pedagogical Content Knowledge (TPACK), an educational product was developed - a dynamic book built on the book option on the GeoGebra website -

\footnotetext{
${ }^{1}$ Mestre em ensino de Ciências, Matemática e Tecnologias pela Universidade do Estado de Santa Catarina (UDESC). Professora universitária na UNISOCIESC e na UDESC; raiane.lemke@gmail.com.

2 Doutora, Professora, Universidade do Estado de Santa Catarina, ivazuchi@gmail.com.
} 
GeoGebraBook, which contains exercises, activities, questions, images, videos, situationproblems and learning objects developed in GeoGebra, organized in an interactive way, with the intention of contributing to the teaching and learning processes of the contents involved.

Keywords: Calculus. GeoGebra. Real functions of two variables.

\section{Introdução}

No contexto do programa de pós-graduação em Ensino de Ciências, Matemática e Tecnologias (mestrado profissional), da Universidade do Estado de Santa Catarina, foi realizada uma pesquisa qualitativa, sobre o Ensino de Cálculo, principalmente do conteúdo de funções reais de duas variáveis reais (F2V) e alguns tópicos de derivadas parciais, como interpretação geométrica e taxa de variação (LEMKE, 2017). Com base em outras pesquisas averiguou-se uma problemática no ensino e aprendizagem dos conteúdos sobreditos.

Desse modo, pensando na temática da visualização, utilizando o software GeoGebra $^{3}$ e considerando o quadro teórico do Conhecimento Tecnológico e Pedagógico do Conteúdo (TPACK) ${ }^{4}$, foi desenvolvido um produto educacional cujo título é "Funções reais de duas variáveis e GeoGebraBook: recursos dinâmicos para o ensino de Cálculo5". Um GeoGebraBook é um tipo de livro online, sendo o produto educacional um material interativo, que contém propostas de atividades, questionamentos, figuras, vídeos, situações-problemas e objetos de aprendizagem desenvolvidos no GeoGebra, dentre outros recursos, com o objetivo de proporcionar uma abordagem dinâmica para ensinar Cálculo, tendo como público professores e alunos de Cálculo de funções de várias variáveis.

Ademais, apresentaremos o produto educacional com mais detalhes, evidenciando sua relevância, seus objetivos, sugestões de uso, suas potencialidades e limitações para utilização em sala de aula, bem como, sucintamente, a metodologia e fundamentação teórica envolvida.

${ }^{3}$ Software de geometria dinâmica que combina conceitos de geometria e álgebra. Disponível em: $<$ https://www.geogebra.org >. Acesso em: 17 jan. 2018.

4 Do inglês, Technological Pedagogical Content Knowledge. Concebido por Mishra e Koehler (2006), Koehler e Mishra (2008).

${ }^{5}$ Disponível em: <https://ggbm.at/GdZ9wzW8>. Acesso em: 20 abr. 2018. 


\section{Fundamentação teórica}

Permearam na elaboração do produto educacional o ensino de Cálculo, a visualização, o uso de softwares de geometria dinâmica (SGD) e o Conhecimento Tecnológico e Pedagógico do Conteúdo (TPACK).

Optamos em abordar o ensino de Cálculo tendo em vista que essa disciplina, em geral, apresenta altos índices de reprovações e dificuldades dos alunos, conforme comentam os pesquisadores Rezende (2003), Imafuku (2008), Machado, R. (2008), Aguiar e Moro (2013) e Oliveira (2014). Mais especificamente, escolhemos investigar sobre o ensino de Cálculo a várias variáveis (CVV), pois ainda são poucos os estudos que abordam o ensino de CVV e corroborando com Alves; Borges e Machado (2007) são escassos os trabalhos sobre a noção de derivada em várias variáveis.

No ensino de CVV a visualização exerce um papel considerável para a compreensão de diversos conteúdos, como problemas de derivadas parciais e algumas de suas aplicações. A visualização, como aponta Oliveira (2014), é uma das dificuldades apresentadas pelos alunos de CVV. A matemática pode ser difícil e abstrata de ser compreendida, porém, conforme Machado (2008), com o uso de computadores, por exemplo, os alunos terão a possibilidade de calcular, visualizar e muitas vezes manipular objetos e operações que, sem tais recursos, seriam inimagináveis. A partir de nossas leituras e de nossas experiências ponderamos que as tecnologias, como softwares de geometria dinâmica (SGD), podem ser bons recursos para o ensino de funções de duas variáveis. Desse modo, na construção do produto educacional, buscamos por oferecer recursos que auxiliassem na visualização dos conceitos matemáticos envolvidos.

Dentre os SGD optamos em trabalhar com o GeoGebra, utilizando a versão GeoGebra 5.0 que possui recursos tridimensionais. Quanto ao uso do GeoGebra em sala de aula entendemos que não basta apenas o professor saber manipular a ferramenta, além disso é necessário que o professor tenha o domínio do conteúdo e tenha uma preocupação pedagógica, pensando em como a utilização do GeoGebra pode ajudar na aprendizagem. O vínculo entre esses três aspectos, o 
conhecimento tecnológico, o conhecimento do conteúdo e o conhecimento pedagógico, está presente no quadro teórico concebido por Mishra e Koehler (2006), Koehler e Mishra (2008), denominado TPACK - "Conhecimento Tecnológico e Pedagógico do Conteúdo". O TPACK também se fez presente no desenvolvimento dos objetos de aprendizagem $(\mathrm{OAs})^{6}$, pois utilizamos conhecimentos tecnológicos - como o domínio do software GeoGebra, conhecimento do conteúdo - no caso um conteúdo de matemática, tal como F2V, e conhecimento pedagógico, quando pensamos na maneira como os OAs poderiam ser aplicados no ensino.

Como surgiu a ideia do produto educacional a partir da pesquisa realizada? Como o produto educacional foi idealizado? Quais são suas limitações? Como pode ser utilizado e adaptado em ambientes educacionais? Questões como essas serão discutidas a seguir.

\section{Criação do Produto Educacional e sugestões de utilização}

A partir da pesquisa realizada (LEMKE, 2017) constatamos dificuldades na aprendizagem de CVV e sentimos a necessidade de oferecer um recurso que possibilitasse uma visualização dos conceitos matemáticos envolvidos, utilizando um SGD. Além disso, pensamos em organizar o produto educacional proporcionando uma maneabilidade simples e interativa.

A ideia principal já estava definida - construir recursos dinâmicos que favorecessem a visualização e compreensão dos conceitos pertinentes, mais especificamente no que diz respeito à $\mathrm{F} 2 \mathrm{~V}$, derivadas parciais e algumas de suas aplicações. A questão estava em como organizar esses materiais. Cogitamos a possibilidade de fazer na forma impressa, no entanto, isso exigiria mais tempo e custo, principalmente devido à edição e diagramação, além de oferecer um acesso mais restrito em relação às possibilidades de um material digital. Assim,

\footnotetext{
6 Entendemos, em consonância com Santos (2007), como qualquer recurso como maquetes, fotos, imagens, vídeos, arquivos de texto, páginas de internet, quando utilizadas como recursos que apoiam processos de ensino e aprendizagem.
} 
pensamos em propor um e-book. Foram examinadas algumas ferramentas de publicação online, tais como Issuu e wix, porém foram descartadas por não favorecem a escrita de equações matemáticas. Investigando constatou-se que o próprio site do GeoGebra oferecia o recurso que procurávamos, o chamado GeoGebraBook, no qual pudemos inserir textos, equações matemáticas, questões de múltipla escolha, imagens, materiais do GeoGebra, links, vídeos, arquivos em pdf e slides em PowerPoint, tudo isso organizado de maneira interativa.

O produto educacional pode ser utilizado da forma como está disponível no link $^{7}$, podendo ser acessado sequencialmente pela ordem dos capítulos e seções, ou de maneira aleatória, ou apenas direcionando-se para o tópico de interesse. Para poder navegar pelo produto educacional é necessário ter acesso à rede.

Apesar de ser compatível com tablets e smartphones, recomendamos que seu acesso seja feito em um computador ou notebook, pois a visualização e manuseio são mais adequados. Além disso, o professor pode projetar o livro, propor que os alunos acessem o livro extraclasse ou realizar atividades durante a aula em um laboratório de informática no qual cada aluno ou dupla de alunos tenham acesso à computadores conectados à rede.

De uma maneira geral, o produto educacional pode ser utilizado no momento da aula ou proposto como atividade extraclasse. Pode ser usado para introduzir o conteúdo, como revisão ou como recursos para ampliar a discussão sobre o conteúdo.

Um conteúdo abordado em Cálculo 2 e que se faz presente em nosso produto educacional são funções reais de duas variáveis reais (F2V). O professor pode utilizar o capítulo que contém aplicações de F2V para introduzir o conteúdo, discutindo por exemplo, que o índice de massa corporal (IMC) de uma pessoa pode ser descrito como uma função que depende de duas variáveis: peso e altura. Este capítulo também pode ser indicado pelo professor como leitura complementar extraclasse para os alunos, ou ainda, ser discutido durante a aula, depois de já ter definido F2V.

\footnotetext{
${ }^{7}$ Disponível em: <https://ggbm.at/GdZ9wzW8>. Acesso em: 10 jan. 2018.
} 
Portanto, a utilização do produto educacional é versátil, dependendo dos interesses e intenções dos professores e alunos. Além disso, possuindo uma conta no GeoGebra é possível copiar o produto educacional, podendo ser editado e adaptado. A Figura 1 ilustra essa possibilidade.

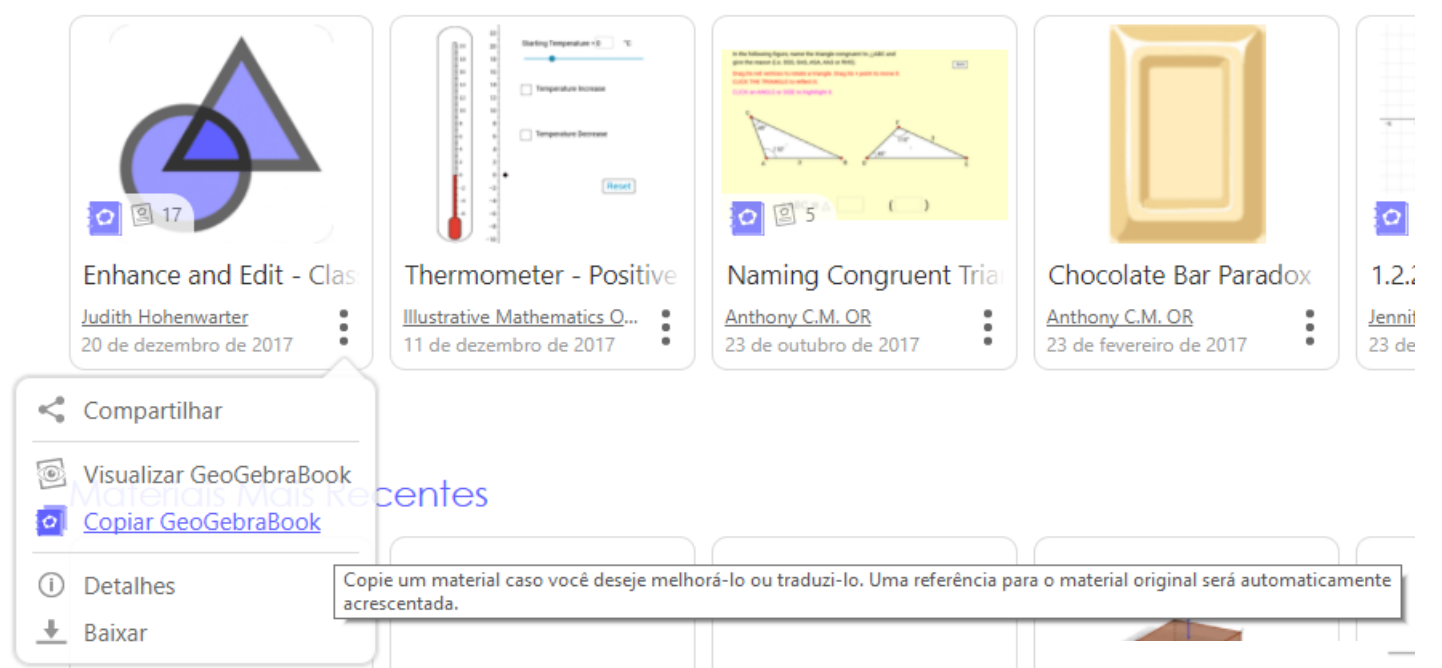

Figura 1: Como copiar um GeoGebraBook para editá-lo.

Fonte: Produção própria, 2018.

Como limitações do produto educacional temos que ele é uma material online, então sem conexão à rede não é possível acessá-lo de maneira interativa. Apesar disso, o professor ou aluno pode baixar alguns itens do produto educacional, tais como OAs, e usá-los off-line, desde que se tenha o GeoGebra instalado. A Figura 2 ilustra a opção de download de um material do GeoGebra.

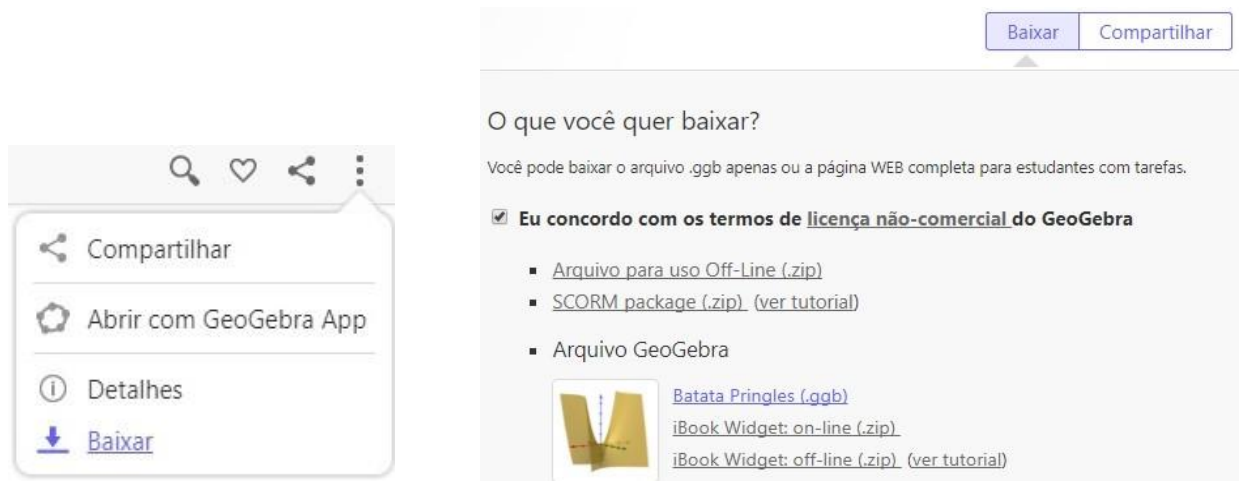

Figura 2: Download de um objeto de aprendizagem desenvolvido no GeoGebra. Fonte: Produção própria, 2018. 
Outra limitação pode ser a projeção de OAs no GeoGebra utilizando um Datashow. Não fizemos uma verificação detalhada dos contrastes entre as cores de fundo e das caixas de texto, bem como o tamanho da fonte, podendo assim, a projeção não ficar boa, no entanto, o usuário pode fazer o download do OA desejado e editar as cores e tamanho das letras.

\section{Apresentação do Produto Educacional}

Para a organização do produto educacional, utilizamos a opção de livro online oferecida no site do GeoGebra, chamado de GeoGebraBook ${ }^{8}$, no qual é possível inserir diversos recursos, como ilustrado na Figura 3. O uso dessa ferramenta é gratuito, sendo necessário apenas criar uma conta para ter acesso.

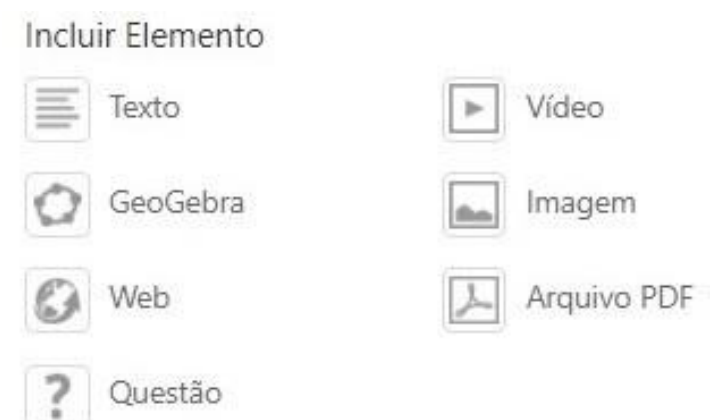

Figura 3: Recursos do GeoGebraBook. Fonte: Produção própria, 2018.

O produto educacional é destinado a professores e alunos de Cálculo de funções de várias variáveis. Pensando em propor abordagens diferenciadas para contribuir para o ensino de Cálculo, especificamente no que tange às funções reais de duas variáveis reais (F2V) e derivadas parciais, desenvolvemos um GeoGebraBook intitulado: "F2V: recursos dinâmicos para o Cálculo9".

O produto educacional tem como objetivos específicos representar um ponto, em coordenadas retangulares, no espaço tridimensional; dar exemplos práticos de F2V, mostrar algumas aplicações e construir gráficos; determinar

8 Disponível em: <https://www.geogebra.org/manual/en/The_GeoGebraBook_Editor>. Acesso em: 07 jan. 2018.

${ }^{9}$ Disponível em: <https://ggbm.at/GdZ9wzW8>. Acesso em: 25 abr. 2018. 
curvas de nível à uma superfície; identificar e representar geometricamente o domínio de funções reais de duas e três variáveis; observar paraboloides hiperbólicos em obras arquitetônicas; explorar os coeficientes de um paraboloide hiperbólico; apresentar a garrafa de Klein (uma superfície que normalmente não é abordada nos currículos de Cálculo); relacionar quádricas com F2V; representar geometricamente algumas quádricas. Calcular derivadas parciais; ilustrar geométrica e algebricamente a construção de um plano tangente a uma dada superfície, possibilitando conexões com as derivadas parciais; resolver problemas que envolvam derivadas parciais como taxa de variação; visualizar o aspecto dinâmico do conceito de derivadas parciais como taxa de variação; propor sugestões de avaliações mediadas pelo GeoGebra; relacionar itens do produto educacional com o ensino médio e, por fim, explicar como desenvolver um GeoGebraBook.

O professor ou aluno ao acessar o livro encontrará uma página inicial, conforme ilustrada na Figura 4 e poderá se direcionar ao capítulo desejado.

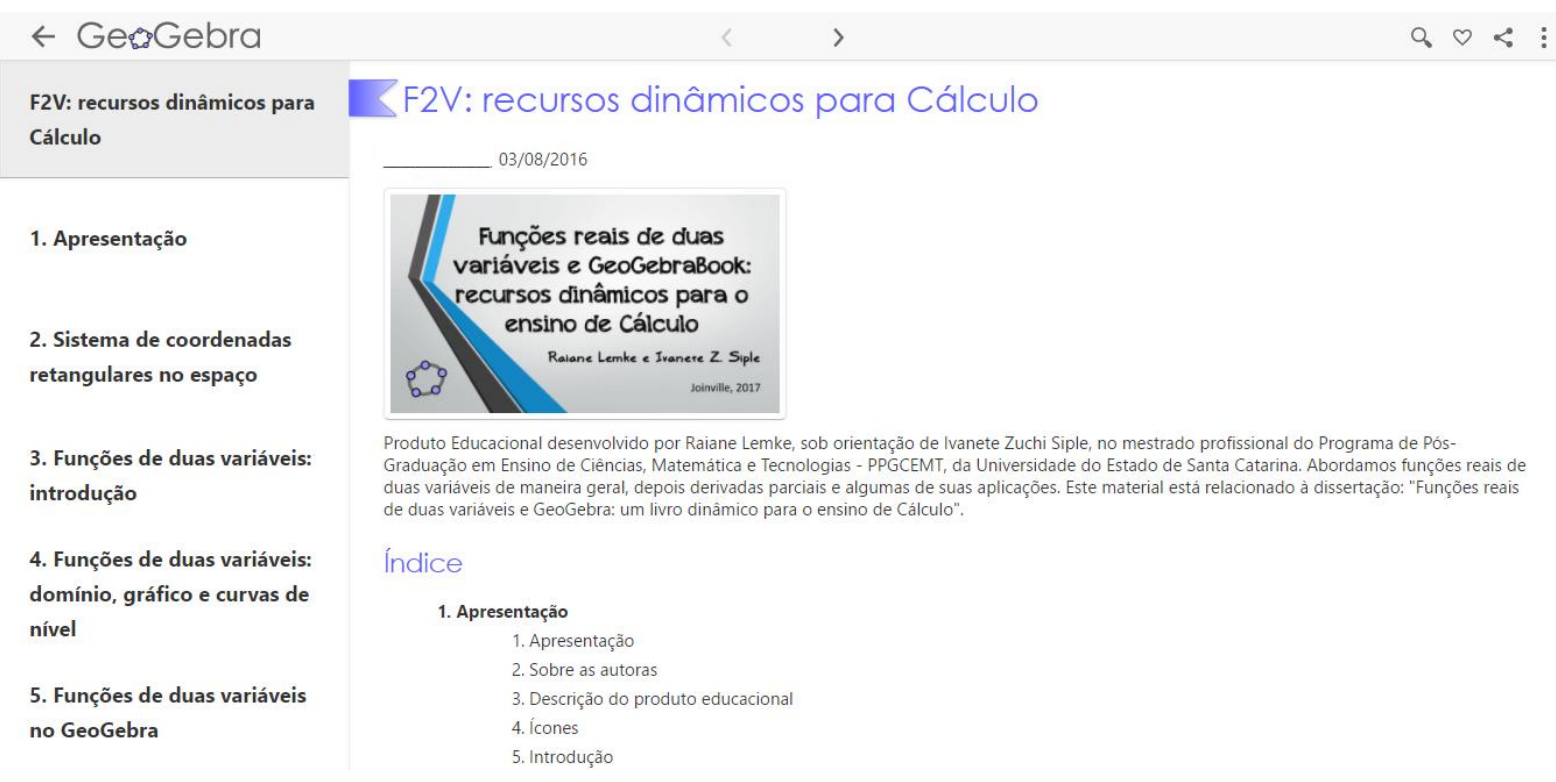

Figura 4: Tela inicial do produto educacional.

Fonte: Produção própria, 2017. 
A maneira de utilizar/explorar os recursos não precisa ser necessariamente feita de forma linear e sequencial, o usuário pode optar pela maneira de navegar no livro. Em termos de elaboração, o produto educacional foi dividido em 18 capítulos. No primeiro capítulo apresentamos a proposta e organização do produto educacional, bem como algumas informações sobre as autoras. Os capítulos 2 a 13 versam sobre funções reais de duas variáveis reais e derivadas parciais. Nos capítulos 14 a 18, propusemos sugestões de avaliações, conexões com o ensino médio, manuais, algumas considerações e perspectivas e um espaço para quem acessar o produto educacional deixar sua opinião.

Num dos capítulos iniciais apresentamos a representação algébrica e geométrica de um ponto no espaço. É um conceito trabalho em Geometria Analítica, mas utilizado em Cálculo 2, pois por exemplo, o gráfico de uma função de duas variáveis pode ser visto como um conjunto de pontos no espaço. Desse modo, este capítulo pode ser utilizado por um professor ou aluno de Cálculo 2 como revisão. Também pode ser utilizado na disciplina de Geometria Analítica, indo além do ambiente bidimensional do lápis e papel ou do quadro e giz, proporcionando uma visualização tridimensional de um ponto no espaço, utilizando o GeoGebra.

O produto educacional contém ícones, com o objetivo de facilitar a visualização das atividades propostas. Como exemplo temos ilustrado, na Figura 5, o capítulo 6 .

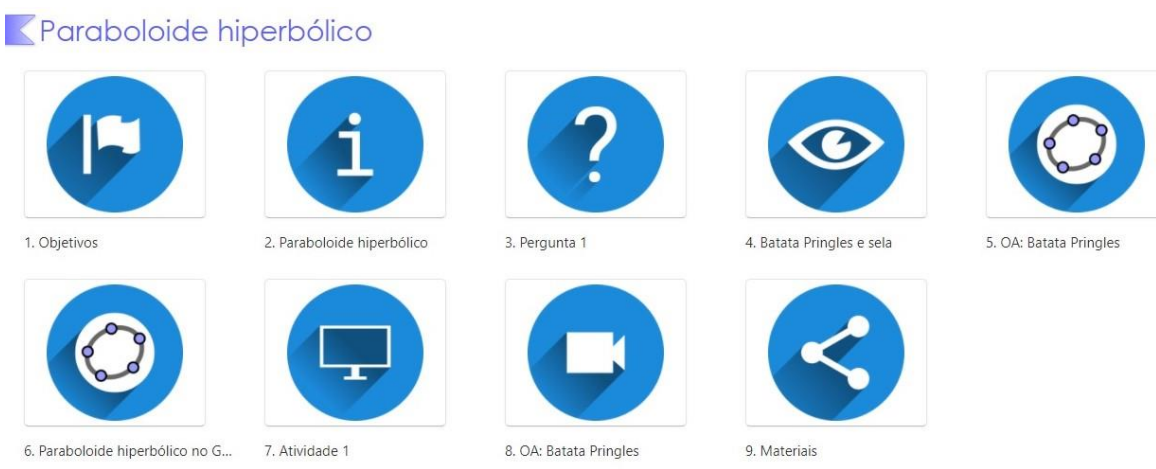

Figura 5: Capítulo 6: Paraboloide hiperbólico.

Fonte: Produção própria, 2018. 
Uma potencialidade do GeoGebraBook que utilizamos em nosso produto educacional é a possibilidade de inserir questões de múltipla escolha que são corrigidas automaticamente. $\mathrm{O}$ usuário seleciona a opção que achar correta e depois clica em verificar respostas, no final da página. Caso a resposta estiver errada, aparecerá um x vermelho na resposta assinalada e a resposta correta ficará destacada em azul. Caso a resposta esteja certa, aparecerá um visto verde, segundo registrado no Quadro 1.

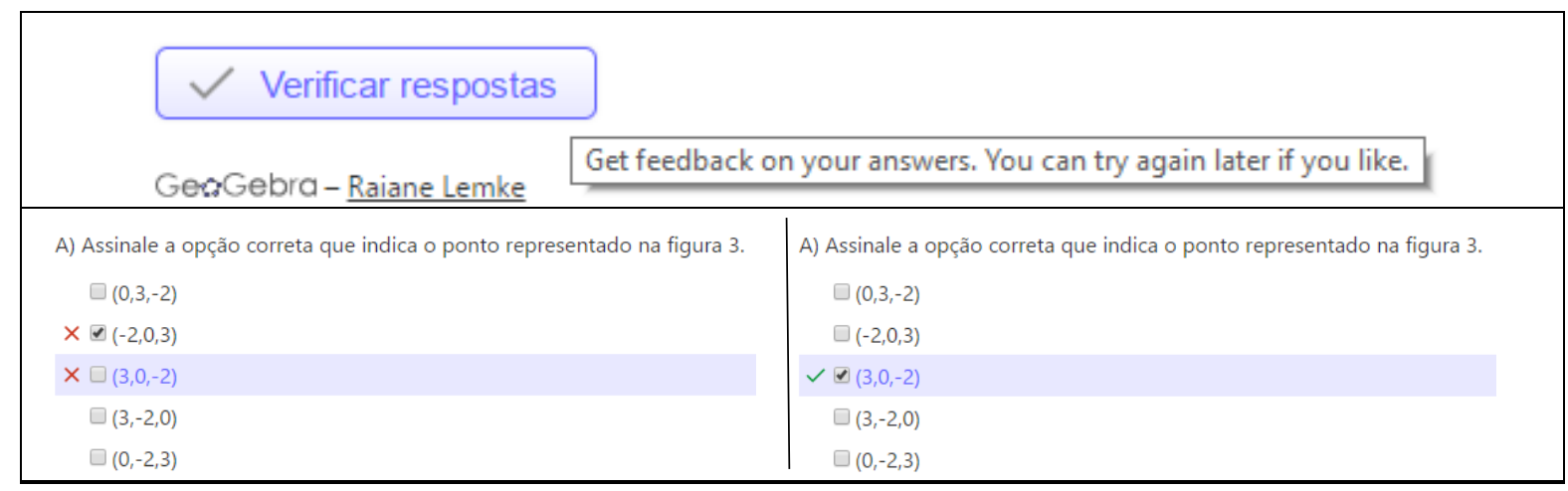

Quadro 1: Feedback em questões de múltipla escolha no GeoGebraBook.

Fonte: Produção própria, 2017.

Como potencialidades para utilização do produto educacional em sala de aula, destacamos sua interatividade de navegação. Além disso, o produto educacional permite que o professor utilize apenas aquilo que ele achar pertinente ou o que the interessar. A intenção do produto educacional é que além de trazer os objetos de aprendizagem (OAs) construídos no GeoGebra, também são apresentados os conteúdos envolvidos, orientações e sugestões de como usar os OAs.

No Quadro 2 listamos os OAs desenvolvidos no GeoGebra que integram o produto educacional. A maioria são de autoria própria, exceto o OA de curvas de nível que foi adaptado de Beier (2016). Os OAs de sistema de coordenadas retangulares, de quádricas e da interpretação geométrica de derivadas parciais foram adaptados de um trabalho de conclusão de curso de graduação - Lemke (2015). 
http://dx.doi.org/10.5965/2357724X06112018018

\begin{tabular}{|c|c|c|}
\hline Imagem & Nome & Disponível em \\
\hline & $\begin{array}{l}\text { Representação do ponto } \\
P(2,3,4)\end{array}$ & https://ggbm.at/AZ2Y7EjX \\
\hline & $\begin{array}{l}\text { Sistema de coordenadas } \\
\text { retangulares no espaço }\end{array}$ & https://ggbm.at/WyXXDmuy \\
\hline & Curvas de nível & https://ggbm.at/gMCWRdkH \\
\hline & Batata Pringles & https://ggbm.at/P8c57SQ3 \\
\hline & Área do retângulo & https://ggbm.at/AUghGkxv \\
\hline & IMC & https://ggbm.at/yw9Yxhpg \\
\hline & Garrafa de Klein: animação & https://ggbm.at/YSUyPuwe \\
\hline & Ponto na garrafa de Klein & https://ggbm.at/aPNcJkX6 \\
\hline & Quádricas centradas & https://ggbm.at/uAHk56pR \\
\hline
\end{tabular}




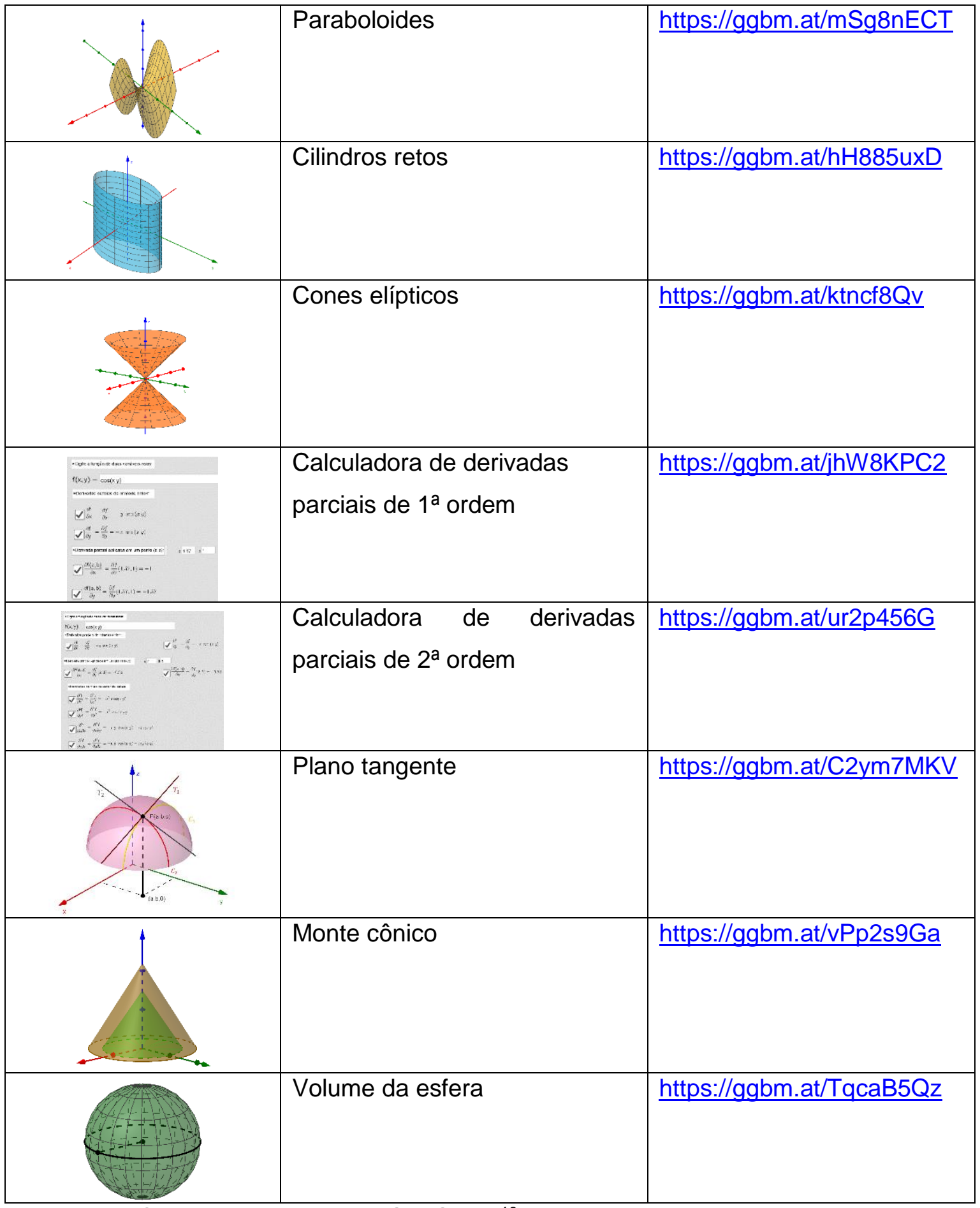

Quadro 2: OAs desenvolvidos no GeoGebra ${ }^{10}$

Fonte: Autor, 2017.

10 Para mais detalhes dos OAs e da estrutura dos capítulos, procure pela descrição do produto educacional "Funções reais de duas variáveis e GeoGebraBook: recursos dinâmicos para o ensino de Cálculo." 
No que segue, destacaremos a relevância do produto educacional, considerando feedbacks recebidos sobre o mesmo e relatando brevemente sobre professores de Cálculo que utilizaram recursos presentes nesse produto educacional.

\section{Relevância do Produto Educacional}

A respeito da formação inicial e continuada de um professor de Matemática, pensamos que o produto educacional possa ser relevante no ensino de Cálculo, pois possibilita recursos diferenciados e dinâmicos, propondo atividades que favoreçam explorar os diferentes registros envolvidos em um determinado objeto matemático.

O produto educacional propriamente dito foi concretizado durante 0 mestrado, em 2017, no entanto, alguns recursos já foram desenvolvidos e aplicados anteriormente, pelas autoras desse trabalho.

Por exemplo, no primeiro semestre de 2015, um professor de Cálculo utilizou o OA do plano tangente em suas turmas de Cálculo 2 para exemplificar a teoria, possibilitando-o superar limitações advindas do uso de recursos como quadro e giz (SIPLE; LEMKE; SANTOS; MANDLER, 2016).

Outra aplicação nesse sentido, ocorreu também no primeiro semestre de 2015, segundo relatam Lemke; Siple e Figueiredo (2016), foram apresentadas potencialidades do GeoGebra e da impressão 3D na criação de OAs para o ensino de funções de várias variáveis. De acordo com Lemke; Siple e Figueiredo (2016) os resultados das aplicações desses OAs têm contribuído no processo de ensino, ajudando na representação gráfica de superfícies, na interseção de superfícies e na visualização de curvas de nível.

Além dessas experimentações, foram realizadas outras durante a pesquisa de mestrado. No segundo semestre de 2016 foi apresentado o protótipo do produto educacional para os integrantes do grupos de pesquisa PEMSA ${ }^{11}$ e

\footnotetext{
11 Grupo de Pesquisa em Educação Matemática e Sistemas Aplicados ao Ensino.
} 
NEPesTEEM. ${ }^{12}$ Estiveram presentes 9 integrantes dos grupos de pesquisa, dentre os quais, 5 professores de Cálculo com mais de dez anos de experiência. Quando solicitados para descrever o produto educacional com uma palavra, obtivemos a nuvem de palavras ilustrada na Figura 6, sendo "dinâmico" uma das palavras mais destacadas.

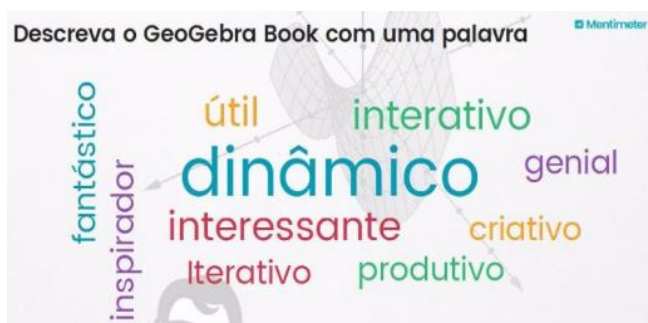

Figura 6: Nuvem de palavras sobre o GeoGebraBook. Fonte: Autor, 2017.

No primeiro semestre de 2017, algumas atividades presentes no produto educacional foram utilizadas por uma professora em sua turma de Cálculo 2 num curso de Engenharia Civil. Foram trabalhados conteúdos como F2V, derivadas parciais, intepretação geométrica de derivadas parciais e derivada parcial como taxa de variação. Ela utilizou esse material para introduzir conceitos, discutir aplicações, investigar representações gráficas, propor atividades e avaliar a aprendizagem dos alunos, conforme relatado em Lemke (2017).

Evidenciamos a nossa proposta de atividade em relacionar obras arquitetônicas com F2V. Utilizando o material proposto (capítulo 3, seção 6), a professora apresentou para os alunos imagens de construções, tais como o pavilhão oceanográfico de Valência, que pode ser modelado por paraboloides hiperbólicos.

Nesse contexto, propusemos uma atividade para que os alunos pesquisassem uma construção que tivesse em sua estrutura o gráfico de uma F2V e redigissem um texto sobre a construção escolhida. Os trabalhos foram tão variados e interessantes que foram organizados de tal modo a ser um capítulo do produto educacional, conforme ilustrado na Figura 7.

\footnotetext{
12 Núcleo de Estudo e Pesquisa em Tecnologia Educacional e Educação Matemática.
} 

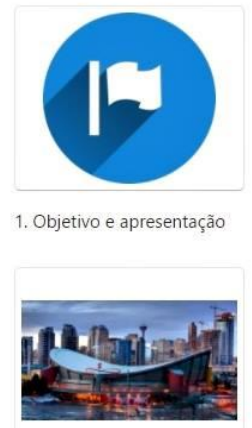

6. Estádio poliesportivo Scotiab..

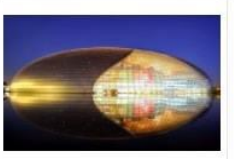

11. Grande Teatro Nacional da C.

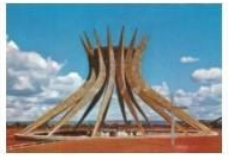

2. Catedral Metropolitana Noss.

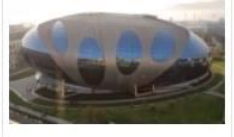

7. Edifício Infosys

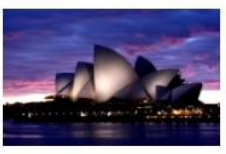

12. Ópera de Sydney

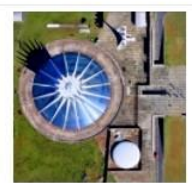

3. Catedral Metropolitana Noss

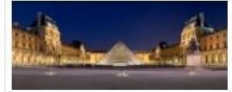

8. Pirâmides do Museu do Louv...

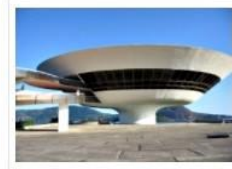

13. Disco Voador de Niterói

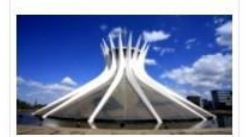

4. Catedral de Brasilia

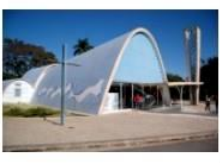

9. Igreja São Francisco de Assis

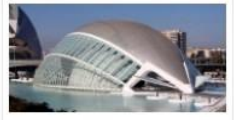

14. Palácio das Artes Rainha Sof.

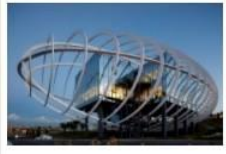

5. Sala de Exposição Tema Istam.

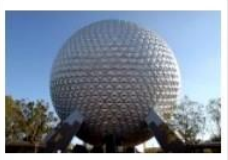

10. Spaceship Earth

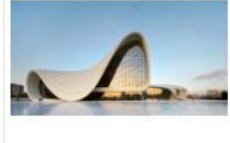

15. Heydar Aliyev Center

Figura 7: Capítulo 10 - Arquitetura e funções de duas variáveis.

Fonte: Autor, 2017.

O produto educacional possui o capítulo "deixe sua opinião", no qual o usuário que acessou o GeoGebraBook pode responder um formulário, deixando comentários, críticas e/ou sugestões sobre o produto educacional. Recebemos alguns feedbacks, dos quais destacamos alguns no Quadro 3.

"Estou perplexa com o material elaborado por vocês. Ele é maravilhoso e de grande potencial para o uso no ensino do cálculo de duas variáveis. Parabéns mil vezes pela qualidade! Usarei sem dúvidas em minhas aulas. Caso surja alguma sugestão encaminho para vocês."

"Parabéns pelo excelente trabalho que vocês realizaram! Sou professora em duas Universidades no RS (UNISINOS e Uergs) e, em ambas, trabalho com o ensino de Cálculo de Várias Variáveis. Já utilizo o GeoGebra na maior parte das minhas aulas por acreditar que a aprendizagem é muito mais significativa quando este recurso é utilizado. Certamente irei sugerir aos meus alunos que acessem este material para estudos extra-classe, pois ficou muito bem estruturado. Obrigada por compartilhar conosco esta pérola!!!! Mais uma vez, parabéns!"

"Gostei muito da proposta, está muito bem organizado. Esta é minha primeira impressão sobre o material, pois, consultei apenas alguns link e explorei poucos OA. Parabéns pelo trabalho!"

Quadro 3: Feedback do produto educacional.

Fonte: Produção própria, 2018. 
Além disso, o produto educacional foi compartilhado em dois grupos do GeoGebra no Facebook, recebendo comentários de professores sobre as suas impressões iniciais do produto educacional proposto e a intenção deles em o utilizarem em suas práticas. Esperamos que o produto educacional proposto, de fato, adentre nas salas de aula e possa contribuir nos processos de ensino e aprendizagem de Cálculo. Também ensejamos, por esses canais, receber dos professores e alunos relatos de suas experiências com esse produto, pois assim, poderemos sempre avaliar e propor evoluções dos recursos propostos.

\section{Considerações}

Proporcionamos um material que possivelmente contribuirá para o ensino de Cálculo tanto pela dinamicidade que ele proporciona como pela visualização, tendo em vista que conceitos, por exemplo, como de taxa de variação, como o próprio nome sugere é um conceito dinâmico e que se somente trabalhado no ambiente do lápis e do papel não permite fazer muitas simulações e experimentações. Uma das potencialidades desse produto educaional é a manipulação dinâmica dos objetos de aprendizagem, articulando diferentes tipos de representações desses objetos.

A integração do conteúdo, tecnologia e pedagogia pode propiciar práticas inovadoras no ensino de Cálculo. Temos a convicção que apenas a inserção do recurso tecnológico não proporciona uma nova prática, sendo assim necessária uma reflexão de como os recursos propostos podem ser aplicados em sala de aula de forma a potencializar a aprendizagem dos alunos. Entretanto, muitas vezes o professor não utiliza a tecnologia em sala de aula por desconhecer a ferramenta. Assim, no produto educacional foram disponibilizados manuais do GeoGebra de uma forma geral, instruções sobre o produto educacional e dicas de como fazer um GeoGebraBook, bem como sugestões de aplicações.

Cabe ressaltar que o produto educacional, na versão on-line, não é algo fechado, mas que poderá passar por melhorias, atualizações e modificações, devido às contribuições de pessoas que colocarem em prática o produto 
educacional, lembrando inclusive que há um espaço no próprio produto educacional (capítulo 18) no qual o professor ou o aluno podem compartilhar experiências sobre sua utilização. Como exemplo, a partir das experimentações realizadas, surgiram novas adaptações que estão sendo sempre implementadas; como sugestões de modificações nos textos, na apresentação dos OAs, sugestões de novos OAs. Também, o produto educacional, disponibilizado on-line recentemente, já recebeu alguns feedbacks positivos no que diz respeito à organização do material e o interesse e intenção, da parte de alguns professores, em usar o produto educacional em suas aulas.

Frisamos que o produto educacional desenvolvido está em constante processo de evolução. Tal evolução é oriunda tanto dos feedbacks das experimentações realizadas em sala de aula quanto da própria evolução das ferramentas. Desejamos que ele possa ter um impacto em sua prática ou em sua aprendizagem e que os resultados oriundos delas possam contribuir na evolução do próprio produto.

\section{Agradecimentos}

Os autores agradecem a FAPESC - Fundação de Amparo à Pesquisa e Inovação do Estado de Santa Catarina pelo apoio financeiro ao Grupo de Pesquisa PEMSA.

\section{Referências}

AGUIAR, Rogério de; MORO, Graciela. Reflexões sobre o ensino de derivada e diferencial de funções de duas variáveis nos cursos de ciências exatas. In: XI Encontro Nacional de Educação Matemática, 2013, Curitiba. Anais eletrônicos... Curitiba: SBEM, 2013. Disponível em:

<http://sbem.web1471.kinghost.net/anais/XIENEM/pdf/1247_340_ID.pdf> Acesso em: 04 jun. 2017.

ALVES, Francisco Regis Vieira; BORGES NETO, Hermínio; MACHADO, Rosélia Costa de Castro. Uma sequência de ensino para a aquisição do conceito de derivadas parciais, direcionais e teoremas correlatos no Cálculo em Várias Variáveis. In: Conexões: Ciência e Tecnologia, Ceará, v. 1, n.1, 2007. Disponível em: <http://conexoes.ifce.edu.br/index.php/conexoes/article/view/137/128> Acesso em: 06 jun. 2017. 
BEIER, Gustav Eckard Gorniski. Curvas de Nível. 2016. Disponível em: <https://ggbm.at/TySN6895>. Acesso em: 07 jun. 2017.

IMAFUKU, Roberto Seide. Sobre a passagem do estudo de uma variável real para o caso de duas variáveis. Dissertação (Mestrado em Educação Matemática) - São Paulo, Pontifícia Universidade Católica de São Paulo, 2008.

KOEHLER, Matthew J., MISHRA, Punya. Introducing Technological Pedagogical Knowledge. In: Annual Meeting of the American Educational Research Association, New York City, March 24-28. 2008. Disponível em: < http://punya.educ.msu.edu/presentations/AERA2008/MishraKoehler_AERA2008.pdf > Acesso em: 19 mai. 2016.

LEMKE, Raiane. Objetos de aprendizagem para o ensino de funções de duas variáveis: um diferencial dinâmico. 2015. TCC (Licenciatura em Matemática) Universidade do Estado de Santa Catarina, Joinville, 2015.

LEMKE, Raiane; SIPLE, Ivanete Zuchi; FIGUEIREDO, Elisandra Bar de. OAs para o Ensino de Cálculo: potencialidades de tecnologias 3D. In: Revista Novas Tecnologias na Educação (RENOTE), v.14 n.1, 2016.

LEMKE, Raiane. Funções reais de duas variáveis e GeoGebra: um livro dinâmico para o ensino de Cálculo. 2017. Dissertação (Mestrado Profissional em Ensino de Ciências, Matemática e Tecnologias) - Universidade do Estado de Santa Catarina, Joinville, 2017.

MACHADO, Rosa Maria. A Visualização na resolução de problemas de Cálculo Diferencial e Integral no ambiente computacional MPP. 2008. Tese (Doutorado em Educação) - Universidade Estadual de Campinas, Campinas, 2008.

MISHRA, Punya, KOEHLER, Matthew J. Technological Pedagogical Content Knowledge: A framework for teacher knowledge. In: Teachers College Record, v. 108, n.6, p. 10171054, 2006. Disponível em:

<http://punya.educ.msu.edu/publications/journal_articles/mishra-koehler-tcr2006.pdf>. Acesso em: 25 jul. 2016.

OLIVEIRA, Fabio Luiz de. A Produção de Conhecimento Matemático acerca de Funções de Duas Variáveis em um Coletivo de Seres-humanos-com-mídias. 2014. Dissertação (Mestrado em Educação Matemática) - Universidade Federal de Ouro Preto, Ouro Preto, 2014.

REZENDE, Wanderley Moura. O ensino de Cálculo: dificuldades de natureza epistemológica. 2003. Tese (Doutorado em Educação) - Universidade de São Paulo, São Paulo, 2003.

SANTOS, Luciane Mulazani. Produção de significados para objetos de aprendizagem: de autores e leitores para a educação matemática. Dissertação (Mestrado em Educação) - Universidade Federal do Paraná, Curitiba, 2007. 
http://dx.doi.org/10.5965/2357724X06112018018

SIPLE, Ivanete Z.; LEMKE, Raiane; SANTOS, Luciane M.; MANDLER, Marnei L. TIC na prática docente: o olhar de um professor de cálculo diferencial e integral. Revista Docência do Ensino Superior, v. 6, p. 115-134, 2016. 